\title{
La relación de Helicobacter pylori con la displasia y el cáncer gástrico en Costa Rica
}

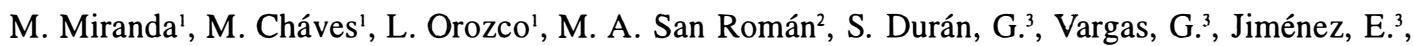 \\ Peña,. L. ${ }^{3}$, Rodríguez, L. ${ }^{3}$ y. E. Barrantes ${ }^{2}$ \\ Servicio de Gastroenterología, Hospital San Juan de Dios. San José, Costa Rica. \\ Laboratorio Clínico, Hospital San Juan de Dios San José, Costa Rica. \\ Servicio de Patología, Hospital San Juan de Dios San José, Costa Rica.
}

\begin{abstract}
Occurrence of the bacterium Helicobacter pylori was compared for two Costa Rican sites with contrasting levels of gastric cancer incidence, Poás (incidence 15. 13\%) and Puriscal (83. 53\%). A sample of 185 adults of similar age and sex proportions was studied in each site, using both $H$. pylori antiserum tests and gastroscopy to collect two biopsies per case. No clear association between $H$. pylori and gastric cancer was found.
\end{abstract}

Key words: Gastric cancer, associations, causes, tropics.

Costa Rica es un país con una incidencia alta de cáncer gástrico; estudios previos han demostrado que hay $46.9 \%$ en hombres y $21.3 \%$ en mujeres (Anónimo 1992). La incidencia cantonal tiene marcadísimas variaciones (Miranda et al. 1977, Sierra y Barrantes 1983). Sin embargo, las causas determinantes de la alta incidencia en Costa Rica y sus variaciones no han sido establecidas.

El descubrimiento del Helicobacter pylori ha producido una verdadera revolución en el campo de la gastroenterología en la última década (Marshall 1987). En la actualidad se considera esta bacteria como un agente causal de la gastritis crónica antral (Dixon 1988). Esta entidad, especialmente la forma atrófica, constituye un factor de riesgo del cáncer gástrico, por lo que se piensa que el $H$. pylori puede estar involucrado en la carcinogénesis gástrica.
En poblaciones con riesgo alto de cáncer gástrico se han observado tasas altas de infección con H. pylori (Forman et al. 1990, Correa et al. 1990, Burstein et al. 1991, Anónimo 1993).

En otras áreas con incidencia alta de H. pylo$r i$, se han encontrado tasas bajas de cáncer gástrico (Holcombe 1992) y en ciertos grupos de pacientes no se ha podido establecer una relación directa entre el cáncer gástrico y la infección por H. pylori (Farinati et al. 1993, Kwipers et al. 1993).

Por otra parte, está bien establecido que la gastritis crónica atrófica, la metaplasia intestinal y la displasia de la mucosa gástrica, constituyen lesiones pre-neoplásicas de la mucosa gástrica y su frecuencia es paralela al riesgo de cáncer gástrico en cada región (Correa et al. 1976). 
El presente trabajo se realizó con el fin de establecer la relación de la infección con $H$. pylo$r i$, las lesiones pre-neoplásicas del estómago y el cáncer gástrico en dos cantones (Poás y Puriscal) de incidencia contrastada de cáncer gástrico en Costa Rica.

\section{MATERIALES Y METODOS}

El presente estudio se hizo en Costa Rica, con el objetivo de comparar un cantón de muy baja incidencia de cáncer gástrico, como el de Poás (incidencia para ambos sexos de 15.13\%) (Miranda et al. 1977), con otro de incidencia muy alta, Puriscal (incidencia para ambos sexos de 83. 53\%) (Miranda et al. 1977). El estudio se hizo en las Clínicas del Seguro Social en personas nacidas y residentes en estos cantones, ya fueran referidos por sintomatología digestiva, y en voluntarios asintomáticos. Se escogieron al azar 185 adultos de cada cantón, similares en edad y sexo. En ellos se practicó un estudio serológico para buscar anticuerpos IgG para $H$. pylori, utilizando el enzimoinmunoanálisis HM-CAP (Enteric Products Inc. Westbury, N. Y. ). La sensibilidad y especificidad de este método es de $98.7 \%$ y $100 \%$ respectivamente. Los sueros fueron diluidos 1: $100 \mathrm{y}$ analizados de acuerdo a las instrucciones de fabricante, en el Laboratorio Clínico de Hospital San Juan de Dios.

En todos los pacientes se practicó una gastroscopía utilizando un equipo Olympus GIF Q20 y se tomaron dos biopsias, una del antro y otra del fondo utilizando pinzas del modelo FB$24 \mathrm{~K}$, que produce una muestra de tejido de 2 $\mathrm{mm}$ de diámetro. Las biopsias fueron incluidas en parafina y seccionadas a 2 y 5 micras y teñidas con hematoxilina-eosina, PAS, azul de Alsacia y solución Diff Quick $\mathrm{N}^{\circ} 2$, para demostrar la presencia de $H$. pylori en la mucosa gástrica. Las biopsias fueron procesadas y examinadas en el Laboratorio de Patología del Hospital San Juan de Dios, sin información de los antecedentes clínicos.

Se aplicaron los criterios histológicos conocidos para clasificar los tejidos como normal, con diversos grados de gastritis crónica, con metaplasia intestinal, con displasia o con cáncer.

El diagnóstico de gastritis por $H$. pylori se estableció cuando la mucosa gástrica presentaba infiltrado crónico, al que puede agregarse infiltraciones por polimorfonucleares y presencia de folículos linfáticos en el corion o la submucosa. $H$. pylori se observa en grupos de bacilos fusoespirilares en el mucus que recubre la mucosa o en las fóveas gladulares.

Los pacientes objeto de este estudio forman parte de grupos mayores de los cantones de Poás y Puriscal en los cuales se realizan otros estudios, como parte del proyecto CCSS-Clubes Rotarios-Asociación Costarricense de Gastroenterología.

\section{RESULTADOS}

Los resultados se exponen en el Cuadro 1.

La positividad serológica por $H$. pylori es similar en ambos cantones. La gastritis por $H$. pylori es es más alta en el cantón de muy bajo riesgo (Poás), que en el de muy alto riesgo (Puriscal). La metaplasia intestinal tiene frecuencia similar en ambos sitios. La displasia es significativamente más frecuente en Puriscal. Dos casos de cáncer temprano se encontraron en Puriscal y ninguno en Poás, pero este dato no es significativo.

\section{DISCUSIÓN}

Tradicionalmente, las grandes diferencias en la incidencia de cáncer gástrico que existen en el mundo eran explicadas por variaciones en la dieta y el estatus socioeconómico (Cuello et al. 1982). También, en forma reiterada, los estudios dietéticos han demostrado que en las zonas de alto riesgo hay bajo consumo de frutas y verduras frescas (Correa 1992).

Sin embargo, el estudio de la acción de $H$. pylori ha cambiado este esquema y se ha postulado que esta bacteria es responsable de la producción de úlcera gástrica y duodenal y puede 


\section{CUADRO 1}

Resultados del estudio de 185 pacientes de los cantones de Poás y Puriscal.

\begin{tabular}{|c|c|c|c|}
\hline & Poás & Puriscal & Significancia \\
\hline $\begin{array}{c}\text { Serología Positiva } \\
\text { para } H \text {. pylori }\end{array}$ & $151(82 \%)$ & $153(83 \%)$ & N.S. \\
\hline $\begin{array}{l}\text { Histología normal } \\
\text { en fondo } 32\end{array}$ & $\begin{array}{l}\text { en antro } 26 \\
\text { en fondo } 70\end{array}$ & $\begin{array}{c}\text { en antro } 20 \\
<.0001\end{array}$ & N.S \\
\hline $\begin{array}{c}\text { Gastritis por } \\
\text { H. pylori }\end{array}$ & $\begin{array}{l}\text { en antro } 115 \\
\text { en fondo } 29\end{array}$ & $\begin{array}{l}\text { en antro } 88 \\
\text { en fondo } 15\end{array}$ & $\begin{array}{l}<.0065 \\
<.0400\end{array}$ \\
\hline $\begin{array}{l}\text { Metaplasia } \\
\text { intestinal }\end{array}$ & $\begin{array}{l}\text { en antro } 17 \\
\text { en fondo } 0\end{array}$ & $\begin{array}{l}\text { en antro } 16 \\
\text { en fondo } 2\end{array}$ & $\begin{array}{l}\text { N.S } \\
\text { N.S }\end{array}$ \\
\hline $\begin{array}{l}\text { Displasia } \\
\text { en fondo } 0\end{array}$ & $\begin{array}{l}\text { en antro } 1 \\
\text { en fondo } 0\end{array}$ & $\begin{array}{c}\text { en antro } 11 \\
\text { N.S }\end{array}$ & $<.0056$ \\
\hline Cáncer temprano & en antro 0 & en antro 2 & N. S. \\
\hline
\end{tabular}

constituir un factor de riesgo para el cáncer gástrico (Sipponen y Hyvarinen 1993).

Esto último no ha sido definitivamente probado y en una reunión de expertos llevada a cabo en los Institutos Nacionales de Salud de los Estados Unidos a principios de 1994 para valorar el papel que juega $H$. pylori con respecto a su relación con el cáncer gástrico, se concluyó que este aspecto requiere más estudios para probar o descartar esta relación causa-efecto (Anónimo 1994).

Costa Rica ofrece posibilidades muy interesantes para profundizar en el estudio de la relación de $H$. pylori con el cáncer gástrico, al tener bien definidas las zonas de diferente riesgo (Miranda et al. 1977).

Sin embargo, los resultados del presente trabajo, no apoyan la existencia de una fuerte relación entre $H$. pylori y cáncer gástrico. Si así fuera uno esperaría que la positividad de la serología y la gastritis causada por esta bacteria fuera definitivamente más alta en el cantón de alto riesgo, lo que no es así.

En la actualidad estamos llevando a cabo estudios adicionales en otros cantones y con una diferente metodología a fin de perfilar mejor este problema.
En Costa Rica, la displasia gástrica es significativamente más frecuente en el cantón de muy alto riesgo para cáncer gástrico como es Puriscal, que en el cantón de muy bajo riesgo, como Poás. Sin embargo la positividad serológica para $H$. pylori no fue significativamente diferente en ambos cantones y la gastritis por $H$. pylori fue significativamente menos frecuente en el cantón de muy alto riesgo, Puriscal. Estos datos ponen en duda el papel que puede estar jugando la infección por $H$. pylori en el proceso de carcinogénesis gástrica en Costa Rica.

\section{AGRADECIMIENTOS}

Los autores agradecen a la Caja Costarricense del Seguro Social, a los Clubes Rotarios de Costa Rica, a la Asociación Costarricense de Gastroenterología, su auspicio para realizar este trabajo y a Pedro Morera por la revisión y corrección del manuscrito.

\section{RESUMEN}

Se comparó la incidencia de la bacteria Helicobacter pylori en un lugar de baja incidencia de cáncer gástrico, Poás 
(15. 13\%) y otro de incidencia muy alta, Puriscal (83. 53\%) Se eligió a 185 adultos de cada cantón, similares en edad y sexo y se practicó un estudio serológico para buscar anticuerpos IgG para $H$. pylori, y una gastroscopía para tomar dos biopsias por caso. Los resultados no apoyan la existencia de una fuerte relación entre $H$. pylori y cáncer gástrico.

\section{REFERENCIAS}

Anónimo. 1992. Sin título. IARC Sci. publ. 120: 208-209.

Anónimo. 1994. Sin título. AGA News 28: 12-13.

Miranda, M. J. \& L. Macaya M. 1977. Aspectos epidemiológicos del Cáncer Gástrico en Costa Rica. Acta Med. Costarricense 20: 207-214.

Sierra, R. \& R. Barrantes. 1983. Aspectos ecológicos del CáncerGástrico en CostaRica. Rev.Biol.Trop. 31: 17-18.

Marshall, B. J. 1987. History of the discovery of $H$. Pylori, p. 7-23. In M. Blaser, (ed.). Compylobacter pylori in gastritis and peptic ulcer disease. Igaku-Shoin, Nueva York..

Dixon, M. I. 1988. Compylobacter pylori and chronic gastritis, p. 106-116. In B. J. Rathbore \& R. U. Heatley, (eds). Compylobacter pylori and gastroduodenal disease. Blackwell, Oxford.

Forman, D. , T. Silas, D. G. Newell, A. R. Stacey, J. Boreham, R. Peto, T. C. Campbel, J. Li \& J. Chen. 1990. Geographic Association of Helicobacter pylori antibody prevalence and gastric cancer mortality in rural China. Int. J. Cancer 46: 608-611.

Burstein M. , E. Monge, R. León-Banua, R. Lozano, R. Berendson, R. H. Gilman \& C. Rodríguez 1991. Low peptic ulcer and high gastric prevalence in a developing country with a high prevalence on infection by Helicobacter pylori. J. Clin. Gastroenterology 13: 154-156.
Correa P., J. Fox, E. Fontham, B. Ruiz, Y. Lin, D. Zavala, N. Taylor, D. MacKinley, E. De Lima, H. Portilla \& G. Zarama. 1990. Helicobacter pylori and gastric carcinoma. Serum antibody prevalence in populations with contrasting cancer risks. Cancer 66: 2569-2574.

The Eurogast Study Group. 1993. An international association between Helicobacter pylori infection and gastric cancer. Lancet 341: 1359-1362.

Holcombe C. 1992. Helicobacter pylori. The African enigma. Gut. 33: 429-431.

Kwipers E. J. , M. Gracia-Casanova, A. S. Peña, G. Pals, G. Vam Camp, A Kok, A. Kurz-Pohlmann, N. E. Pets \& S. G. Meuwissen. 11993. Helicobacter pylori serology us patients with gastric carcinoma. Scand. J. Gastroenterol. 28: 433-437.

Farinati F. , F. Valiante, R. Germana, G. Della-Libera, R. Baffa, M. Rugge, M. Plebani, F. Vianello, F. Di Mario \& R. Naccarato. 1993. Prevalence of Helicobacter pylori infection in patients with precacerous changes and gastric cancer. Euv. J. Cancer Prew. 2: 231-236.

Correa P. , C. Cuello \& A. Duque. 1976. Gastric Cancer in Colombia. III Natural History of precursor lesions. J. Nat. Cancer Inst. 57: 1027-1035.

Cuello C. , P Correa \& R. W. Haenszel. 1982. Socioeconomic class deferences in cancer incidence in Cali, Colombia. Inst. J. Cancer 29: 637-643.

Correa P. 1992. Human gastric carcinogenesis a multistep and multifactorial process. First American Cancer Society. Award Lecture on Cancer Epidemiology and Prevention. Cancer Res. 52: 634-640.

Sipponen P \& H. Hyvarinen. 1993. Role of Helicobacter pylo$r y$ in the pathogenesis of gastritis, peptic ulcer and gastric cancer. Scand. J. Gastroenterol. 28 (Suppl) 196: 3-61. 